\title{
Substrate specificity of benzamide synthetase involved in 4-hydroxy-3-nitrosobenzamide biosynthesis
}

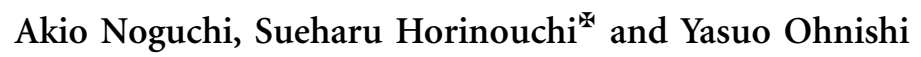 \\ Glutamine-dependent amidotransferase (Gn-AT) catalyzes the transfer of the amido nitrogen of glutamine to an acceptor \\ substrate to produce glutamate and an aminated product. Although most Gn-ATs are involved in biosyntheses of primary \\ metabolites, some are important in the synthesis of secondary metabolites. Recently, a Gn-AT (NspN) was discovered in the \\ biosynthetic pathway of 4-hydroxy-3-nitorosobenzamide in Streptomyces murayamaensis. NspN converts 3-amino-4- \\ hydroxybenzoic acid (3,4-AHBA) to 3-amino-4-hydroxybenzamide. Here, we report the amino-acceptor substrate specificity of \\ NspN. NspN could use several benzoic acid derivatives as amino-acceptor substrates to produce corresponding benzamides and \\ catalyze the amidation of several carboxylate-type phenylpropanoids, such as p-coumaric acid, cinnamic acid and caffeic acid. \\ NspN showed the highest activity toward its natural substrate 3,4-AHBA among the substrates examined. NspN and related \\ bacterial Gn-ATs may be useful in developing combinatorial biosynthetic strategies for benzamide derivatives, which could, in \\ turn, be used as therapeutic agents for a variety of diseases.
}

The Journal of Antibiotics (2011) 64, 93-96; doi:10.1038/ja.2010.144; published online 17 November 2010

Keywords: benzamide synthetase; glutamine-dependent amidotransferase; 4-hydroxy-3-nitrosobenzamide; secondary metabolism; Streptomyces; substrate specificity

\section{INTRODUCTION}

The amido group on the side chain of glutamine is often used as a nitrogen source for biological molecules by glutamine-dependent amidotransferases (Gn-ATs). Gn-ATs catalyze the transfer of the amido nitrogen of glutamine to an acceptor substrate to produce glutamate and an aminated product. This reaction is composed of two half reactions catalyzed by two independent active sites within the enzyme. ${ }^{1,2}$ The first reaction is the hydrolysis of glutamine and the transfer of ammonia to the other active site. The second reaction is the activation of an acceptor substrate and transfer of the ammonia to form the aminated product. Each reaction proceeds in two independent domains of the enzyme, a glutaminase domain for nitrogen transfer at the $N$ terminus and an ATP-dependent synthetase (or synthase) domain for amination at the $C$ terminus. Ammonia travels from the glutaminase domain to the synthetase domain through an ammonia channel. ${ }^{2}$ Gn-ATs are classified primarily into two subfamilies, classes I and II, according to their glutaminase domains. ${ }^{3}$ Glutaminase domains of class I Gn-ATs consist of $\sim 200$ amino-acid residues and have a cysteine protease-type catalytic triad, formed by Cys, His, and Gln. In contrast, class II Gn-ATs have a glutaminase domain in which the $N$-terminal Cys is the only catalytic residue. Although the glutaminase domain uses glutamine as a common nitrogen donor, the synthetase domain exhibits amination activity with a variety of acceptors to produce several metabolites, most of which are primary metabolites, such as amino acids, amino sugars, purine and pyrimidine nucleotides, and coenzymes. There are only a few examples of Gn-ATs that are involved in secondary metabolic processes. $\mathrm{PhzH}$ catalyzes the amidation of phenazine-1carboxylic acid to phenazine-1-carboxamide in the biosynthetic pathway of phenazine-1-carboxamide in Pseudomonas aeruginosa PAO1 ${ }^{4}$ and Pseudomonas chlororaphis PCL1391. ${ }^{5} \mathrm{OxyD}$ and SsfD are involved in the biosynthesis of oxytetracycline in Streptomyces rimosus ${ }^{6}$ and tetracycline SF2575 in Streptomyces sp. SF 2575, ${ }^{7}$ respectively. PcsA and PcsB are polyene carboxamide synthases in the polyene amide biosynthesis pathway in Streptomyces diastaticus ${ }^{8}$ and the AB-400 biosynthetic pathway in Streptomyces RGU5.3, ${ }^{9}$ respectively. TsrC is involved in the biosynthesis of thiostrepton in Streptomyces laurentii. ${ }^{10}$

Recently, we determined the entire biosynthetic pathway of 4-hydroxy3-nitrosobenzamide (4,3-HNBAm) in Streptomyces murayamaensis in which NspN, a class II Gn-AT, plays an essential role. NspN converts 3-amino-4-hydroxybenzoic acid (3,4-AHBA), synthesized from L-aspartic-4-semialdehyde and dihydroxyacetone phosphate by $\mathrm{NspI}$ and $\mathrm{NspH}$, into 3-amino-4-hydroxybenzamide, which is then oxidized by NspF, a novel nitroso-forming $o$-aminophenol oxidase, to yield 4,3-HNBAm (Figure 1). ${ }^{11} \mathrm{NspN}$ amidates 3,4-AHBA, but not 4-hydroxy-3-nitorosobenzoic acid (4,3-HNBA), indicating that

\footnotetext{
Department of Biotechnology, Graduate School of Agriculture and Life Sciences, The University of Tokyo, Tokyo, Japan

Deceased on 12th July 2009.

Correspondence: Professor Y Ohnishi, Department of Biotechnology, Graduate School of Agriculture and Life Sciences, The University of Tokyo, Bunkyo-ku, Tokyo 113-8657, Japan.

E-mail: ayasuo@mail.ecc.u-tokyo.ac.jp

Dedicated to the late $\operatorname{Dr} \mathrm{C}$ Richard Hutchinson for his exceptional contributions to natural product biosynthesis, engineering, and drug discovery.

Received 13 September 2010; revised 25 October 2010; accepted 26 October 2010; published online 17 November 2010
} 


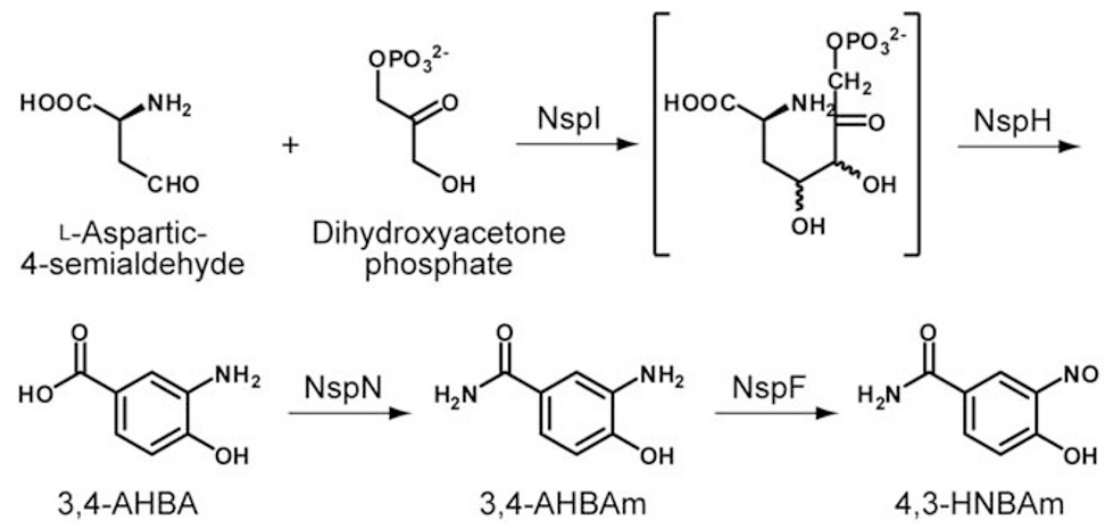

Figure 1 Biosynthetic pathway of 4-hydroxy-3-nitrosobenzamide (4,3-HNBAm). 3-Amino-4-hydroxybenzoic acid (3,4-AHBA) is formed from L-aspartic4-semialdehyde and dihydroxyacetone phosphate by $\mathrm{Nspl}$ and $\mathrm{NspH}$. NspN catalyzes the conversion of 3,4-AHBA to 3-amino-4-hydroxybenzamide (3,4-AHBAm) through amidation of the carboxyl group of 3,4-AHBA. Oxidation of the amino group of 3,4-AHBAm is then catalyzed by NspF to yield 4,3-HNBAm.

amidation precedes oxidation in the conversion of 3,4 -AHBA to 4,3-HNBAm. ${ }^{11}$

The current study further examined the amino-acceptor substrate specificity of NspN. Several benzamide derivatives are used as therapeutic agents for psychiatric diseases (for example, sulpiride, amisulpride, sultopride ${ }^{12}$ and nemonapride ${ }^{13}$ ), as analgesic (for example, salicylamide ${ }^{14}$ ) and as gastrointestinal agents (for example, metoclopramide $^{15}$ and itopride ${ }^{16}$ ). Knowledge of the amino-acceptor substrate specificity of NspN would significantly aid the development of combinatorial biosyntheses using benzamide synthetases.

\section{MATERIALS AND METHODS}

\section{Chemicals}

4-Hydroxy-3-nitrosobenzoic acid ( >94\% purity) was enzymatically synthesized using recombinant $\mathrm{NspF}$ as described previously. ${ }^{11}$ L-Aspartic-4-semialdehyde was synthesized according to the methods described by Black and Wright. ${ }^{17}$ ATP was purchased from Sigma (St Louis, MO, USA). L-Glutamine and L-asparagine were purchased from Nippon Rika, Tokyo, Japan. $\mathrm{MgCl}_{2}$ was purchased from Kokusan Chemical, Tokyo, Japan. Other compounds were purchased from Wako Chemicals, Osaka, Japan.

\section{Enzyme assays of NspN-H activity}

The $\mathrm{His}_{6}$-tag fusion $\mathrm{NspN}$ protein $(\mathrm{NspN}-\mathrm{H})$ was expressed and purified as described previously. ${ }^{11}$ A standard reaction mixture $(50 \mu \mathrm{l})$ consisted of $5 \mathrm{~mm}$ aromatic substrate, $5 \mathrm{~mm}$ ATP, $10 \mathrm{~mm}$ L-glutamine, $10 \mathrm{mM} \mathrm{MgCl}_{2}, 100 \mathrm{~mm}$ HEPES [4-(2-hydroxyethyl) piperazine-1-ethanesulfonic acid sodium salt]$\mathrm{NaOH}$ buffer $(\mathrm{pH} 7.5)$ and NspN-H $(3.3-4.9 \mu \mathrm{g})$. The mixture was preincubated without enzyme for $10 \mathrm{~min}$ at $30^{\circ} \mathrm{C}$, and the reaction was started by addition of the enzyme. After incubation for $60 \mathrm{~min}$ at $30^{\circ} \mathrm{C}$, the reaction was stopped by the addition of $50 \mu \mathrm{l}$ of $5 \%(\mathrm{v} / \mathrm{v})$ trifluoroacetic acid. The reaction products were identified by LC-ESIMS as described below.

A Shodex ODP2 HP-4E column $(4.6 \times 250 \mathrm{~mm}$, Showa Denko K.K., Tokyo, Japan) was used for both HPLC and LC-ESIMS to measure the products of 3,4-AHBA and 4-amino-3-hydroxybenzoic acid catalysis. LC-ESIMS was performed in positive/negative mode on an HPLC system (model 1100 series, Agilent Technologies, Santa Clara, CA, USA) equipped with a mass spectrometer (Bruker HCT plus, Bruker Daltonics, Bremen, Germany). The substrates and products were eluted with a linear gradient of $20-100 \%(\mathrm{v} / \mathrm{v})$ acetonitrile containing $0.1 \%(\mathrm{v} / \mathrm{v})$ acetic acid for $35 \mathrm{~min}$ at a flow rate of $0.3 \mathrm{ml} \mathrm{min}^{-1}$.

The reaction products of the other benzoic acid derivatives and carboxylatetype phenylpropanoids were analyzed on a Senshu Pak DOCOSIL-B columns $(2.0 \times 200 \mathrm{~mm}$ for LC-ESIMS, $3.0 \times 250 \mathrm{~mm}$ for HPLC; Senshu Scientific Co., Tokyo, Japan). LC-ESIMS was performed in positive/negative mode as described above. Both substrates and products were eluted with a linear gradient of $0-100 \%(\mathrm{v} / \mathrm{v})$ acetonitrile containing $0.1 \%(\mathrm{v} / \mathrm{v})$ acetic acid for $25 \mathrm{~min}$ at a flow rate of $0.3 \mathrm{ml} \mathrm{min}^{-1}$.

For enzymatic property analysis, production of 3-amino-4-hydroxybenzamide (3,4-AHBAm) from 3,4-AHBA by the NspN reaction was measured by HPLC. For HPLC, both 3,4-AHBA and 3,4-AHBAm were eluted with a linear gradient of $20-100 \%(\mathrm{v} / \mathrm{v})$ acetonitrile containing $0.1 \%(\mathrm{v} / \mathrm{v})$ acetic acid for $20 \mathrm{~min}$ at a flow rate of $0.5 \mathrm{ml} \mathrm{min}{ }^{-1}$. Eluted compounds were detected by monitoring the elute absorbance at $270 \mathrm{~nm}$ with a L-2450 diode array detector (Hitachi, Tokyo, Japan).

For substrate specificity analysis, the enzymatic activity toward each substrate was measured by a spectrophotometric method. The standard $50-\mu 1$ reaction mixture was incubated for $150 \mathrm{~min}$ at $30^{\circ} \mathrm{C}$, and the reaction was stopped by the addition of $10 \mu \mathrm{l}$ of $5 \mathrm{M} \mathrm{HCl}$. After incubation for $30 \mathrm{~min}$ at room temperature, the mixture was neutralized by the addition of $10 \mu \mathrm{l}$ of $5 \mathrm{M} \mathrm{NaOH}$ and $130 \mu \mathrm{l}$ of $230 \mathrm{~mm}$ HEPES-NaOH ( $\mathrm{pH} 7.5$ ), and mixed with pyrophosphate reagent (Sigma). The presence of pyrophosphate, which was produced from ATP by the NspN reaction, was detected by measuring the solution absorbance at $340 \mathrm{~nm}$. The reaction mixture containing boiled $\mathrm{NspN}-\mathrm{H}$ was also examined as a negative control.

Only a fraction of the total available substrate (less than 10\%) was converted by NspN-H. Thus, the conversion rate was regarded as the apparent activity of $\mathrm{NspN}-\mathrm{H}$ toward each substrate. Each assay was run in triplicate, and the results reported here are mean values.

\section{RESULTS AND DISCUSSION}

\section{Enzymatic properties of NspN}

$\mathrm{NspN}$ is composed of two well-conserved protein motifs commonly associated with asparagine synthetase: a class II glutamine amidotransferase signature in the $N$-terminal glutaminase domain and an asparagine synthetase signature in the $C$-terminal synthetase domain. These features indicate that NspN should use glutamine as an amino donor and that ATP and $\mathrm{Mg}^{2+}$ should be required for the activation of an amino acceptor. To examine the amino-acceptor substrate specificity of NspN, a recombinant $\mathrm{NspN}$ protein $^{11}$ (NspN-H) having a structure of $\mathrm{NspN}^{-H i s_{6}}$ was produced in Streptomyces lividans and purified to homogeneity via metal affinity chromatography and anion exchange chromatography. ${ }^{11}$ As expected, NspN-H showed no activity in the absence of glutamine, ATP or $\mathrm{Mg}^{2+}$, and asparagine could not be used as an amino donor (data not shown). NspN-H was stable at pH $7.5-10$ (at $16^{\circ} \mathrm{C}$ for $18 \mathrm{~h}$ ) and below $30^{\circ} \mathrm{C}$ (at pH 7.5 for $1 \mathrm{~h}$; Figure 2). These ranges are typical for a cytoplasmic enzyme in a mesophilic microorganism. However, the enzymatic activity of 

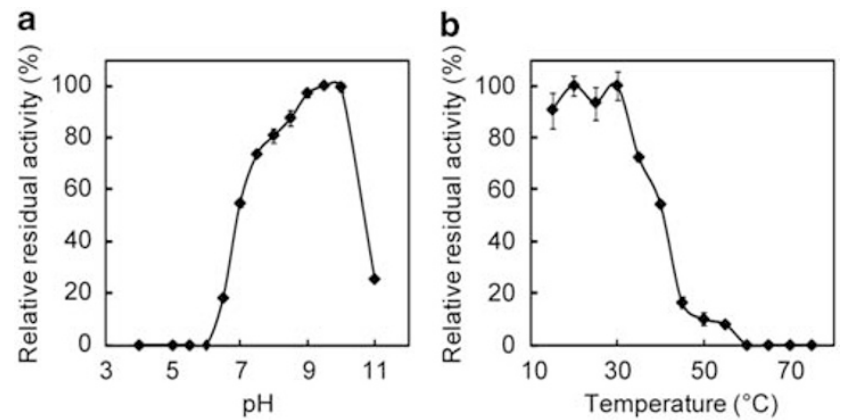

Figure 2 Stability of NspN. The stability of NspN is shown as a function of

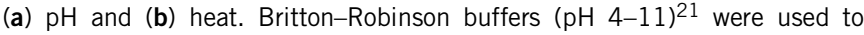
measure NspN activity using a standard assay (see Materials and methods) after storage at $16^{\circ} \mathrm{C}$ for $18 \mathrm{~h}$ in solutions of various $\mathrm{pH}$. For heat stability measurements, $\mathrm{NspN}$ was stored for $1 \mathrm{~h}$ at $\mathrm{pH} 7.5$ at various temperatures and the NspN activity was measured as above.

NspN-H was markedly reduced during purification, which hampered efforts to determine specific activity. Similar observations (a decrease in enzymatic activity during purification) were reported for other Gn-ATs. ${ }^{18-20}$ This decrease in activity was most likely due to oxidation of the free thiol of the catalytic cysteine (Cys-2). However, enzyme activity increased only slightly with increased concentrations of dithiothreitol (up to $5 \mathrm{~mm}$ ) in the reaction buffer.

\section{Substrate specificity of NspN}

The amino-acceptor substrate specificity of NspN was examined by in vitro $\mathrm{NspN}-\mathrm{H}$ reactions using several carboxylic acid derivatives. The reaction products were identified by LC-ESIMS, and the enzyme activity was measured by a spectrophotometric method. The enzymatic activity of $\mathrm{NspN}-\mathrm{H}$ toward various substrates is represented relative to that toward 3,4-AHBA (the most favorite substrate for $\mathrm{NspN}-\mathrm{H}$ ), which was defined as $100 \%$ (Figure 3). NspN-H has been shown to amidate 3,4-AHBA, but not 4,3-HNBA. ${ }^{11}$ Because $3,4-\mathrm{AHBA}$ and 4,3 -HNBA differ only by the functional group $\left(-\mathrm{NH}_{2}\right.$ and $-\mathrm{NO}$, respectively) at the meta position to the $\mathrm{COOH}$ group, benzoic acid derivatives with different functional groups at that position were evaluated as substrates for NspN-H. Amidation of protocatechuic acid (-OH), 4-hydroxy-3-methoxybenzoic acid $\left(-\mathrm{OCH}_{3}\right)$ and $p$-hydroxybenzoic acid $(-\mathrm{H})$ was catalyzed by $\mathrm{NspN}-\mathrm{H}$ with relatively high efficiency (20,32 and 39\%, respectively). Thus, NspN disfavored only 4,3-HNBA among these $m$-substituted $p$-hydroxybenzoic acids. No activity was observed with 5-aminosalicylic acid and $m$-aminobenzoic acid, indicating the importance of the hydroxy group para to the carboxy group in $m$-aminobenzoic acids. No activity was also observed with 4-amino-3-hydroxybenzoic acid. In contrast, the amidation of benzoic acid derivatives that lacked an amino group was catalyzed by NspN-H with relatively high efficiency, regardless of the presence or position of hydroxy group(s). Examples include protocatechuic acid (20\%), 4-hydroxy-3-methoxybenzoic acid (32\%) and p-hydroxybenzoic acid (39\%), all of which are described above, as well as 3-hydroxy-4-methylbenzoic acid (19\%), $m$-hydroxybenzoic acid (8\%), benzoic acid (7\%) and salicylic acid (5\%). Furthermore, NspN-H catalyzed the amidation of some carboxylate-type phenylpropanoids, trans-p-coumaric acid (13\%), trans-cinnamic acid (7\%) and trans-caffeic acid (6\%), although the activities toward these substrates were generally lower than those for the corresponding benzoic acids, p-hydroxybenzoic acid (39\%), benzoic acid (7\%) and protocatechuic acid (20\%), respectively. In

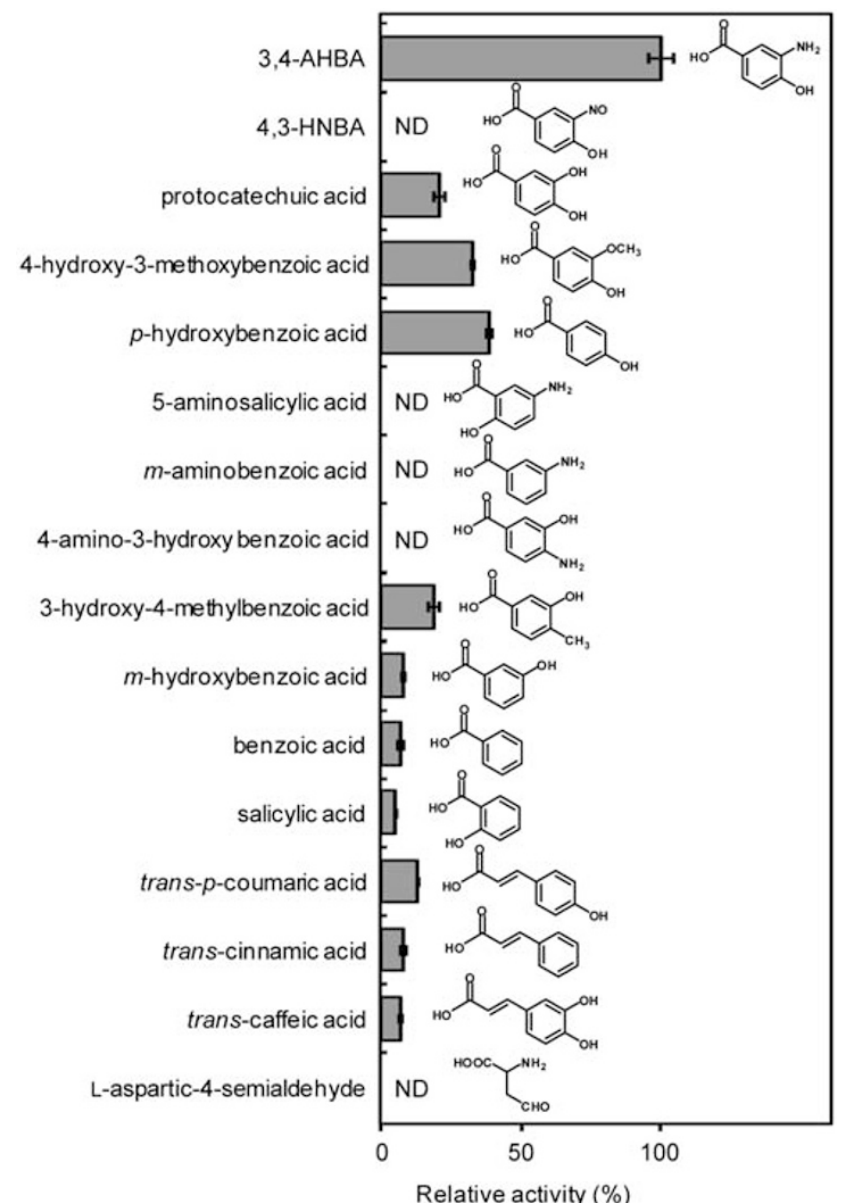

Figure 3 Substrate specificity of $\mathrm{NspN}$. Relative activities of $\mathrm{NspN}$ with benzoic acid and carboxylate-type phenylpropanoid derivatives, and with L-aspartic-4-semialdehyde are shown. Structures of the various substrates are also provided. ND, not detected.

contrast, L-aspartic-4-semialdehyde, a precursor of 3,4-AHBA, was not used as a substrate. This suggests that amidation by NspN occurs with 3,4-AHBA and not with its precursor, L-aspartic-4-semialdehyde, in the 4,3-HNBAm biosynthetic pathway.

\section{Phylogenetic analysis of Gn-ATs}

Protein databases were searched for bacterial Gn-ATs that seem to be involved in secondary metabolism processes, and a phylogenetic tree was constructed using four well-characterized asparagine synthases as the out-group (Supplementary Figure S1 and Supplementary Table S1). This analysis showed that NspN belonged to the same clade as several bacterial Gn-ATs, some of which had been evaluated for their function in secondary metabolite syntheses in vivo. Apart from the clade including NspN, several other bacterial Gn-ATs also seem to be involved in secondary metabolism processes, as suggested by the location of their genes in clusters generally associated with secondary metabolites. Asparagine synthetases, which catalyze the conversion of aspartic acid to asparagine, are phylogenetically distant from NspN.

\section{Conclusions}

NspN-H exhibited a broad amino-acceptor substrate specificity and catalyzed the amidation of (i) several $m$-substituted $p$-hydroxybenzoic acids except 4,3-HNBA, (ii) several benzoic acid derivatives with no amino group (a hydroxy group at the para position to the carboxy 
group was essential for $m$-aminobenzoic acids to be a substrate for NspN-H) and (iii) some carboxylate-type phenylpropanoids, such as trans-p-coumaric acid, trans-cinnamic acid and trans-caffeic acid. NspN-H showed the highest activity toward 3,4-AHBA among the several benzoic acid derivatives and carboxylate-type phenylpropanoids examined in this study. This result is reasonable because 3,4-AHBA is the natural substrate for NspN in 4,3-HNBAm biosynthesis. A phylogenetic analysis of Gn-ATs showed that NspN belongs to a clade of bacterial Gn-ATs that seem to be involved in secondary metabolism processes. NspN and related bacterial Gn-ATs may be useful in combinatorial biosyntheses of benzamide derivatives, which could be used as therapeutic agents for several diseases. This is the first report to describe the amino-acceptor substrate specificity of a bacterial Gn-AT involved in secondary metabolism.

\section{ACKNOWLEDGEMENTS}

This work was supported, in part, by a research grant from the New Energy and Industrial Technology Development Organization of Japan, and by a Grant-inAid for Scientific Research (C) from the Ministry of Education, Culture, Sports, Science, and Technology of Japan (MEXT).

1 Massiere, F. \& Badet-Denisot, M. A. The mechanism of glutamine-dependent amidotransferases. Cell Mol. Life Sci. 54, 205-222 (1998).

2 Mouilleron, S. \& Golinelli-Pimpaneau, B. Conformational changes in ammonia-channeling glutamine amidotransferases. Curr. Opin. Struct. Biol. 17, 653-664 (2007).

3 Schendel, F. J., Mueller, E., Stubbe, J., Shiau, A. \& Smith, J. M. Formylglycinamide ribonucleotide synthetase from Escherichia coli: cloning, sequencing, overproduction, isolation, and characterization. Biochemistry 28, 2459-2471 (1989).

4 Mavrodi, D. V. et al. Functional analysis of genes for biosynthesis of pyocyanin and phenazine-1-carboxamide from Pseudomonas aeruginosa PA01. J. Bacteriol. 183, 6454-6465 (2001).

5 Chin-A-Woeng, T. F., Thomas-Oates, J. E., Lugtenberg, B. J. \& Bloemberg, G. V. Introduction of the phzH gene of Pseudomonas chlororaphis PCL1391 extends the range of biocontrol ability of phenazine-1-carboxylic acid-producing Pseudomonas spp. strains. Mol. Plant. Microbe. Interact. 14, 1006-1015 (2001).

6 Zhang, W., Ames, B. D., Tsai, S. C. \& Tang, Y. Engineered biosynthesis of a novel amidated polyketide, using the malonamyl-specific initiation module from the oxytetracycline polyketide synthase. Appl. Environ. Microbiol. 72, 2573-2580 (2006).

7 Pickens, L. B. et al. Biochemical analysis of the biosynthetic pathway of an anticancer tetracycline SF2575. J. Am. Chem. Soc. 131, 17677-17689 (2009).

8 Seco, E. M., Miranzo, D., Nieto, C. \& Malpartida, F. The pcsA gene from Streptomyces diastaticus var. 108 encodes a polyene carboxamide synthase with broad substrate specificity for polyene amides biosynthesis. Appl. Microbiol. Biotechnol. 85, 1797-1807 (2010).

9 Miranzo, D., Seco, E. M., Cuesta, T. \& Malpartida, F. Isolation and characterization of $p \operatorname{cs} B$, the gene for a polyene carboxamide synthase that tailors pimaricin into $A B-400$. Appl. Microbiol. Biotechnol. 85, 1809-1819 (2010).

10 Liao, R. et al. Thiopeptide biosynthesis featuring ribosomally synthesized precursor peptides and conserved posttranslational modifications. Chem. Biol. 16, 141-147 (2009).

11 Noguchi, A., Kitamura, T., Onaka, H., Horinouchi, S. \& Ohnishi, Y. A copper-containing oxidase catalyzes $C$-nitrosation in nitrosobenzamide biosynthesis. Nat. Chem. Biol. 6, 641-643 (2010).

12 Maitre, M., Ratomponirina, C., Gobaille, S., Hode, Y. \& Hechler, V. Displacement of $\left[{ }^{3} \mathrm{H}\right]$ gamma-hydroxybutyrate binding by benzamide neuroleptics and prochlorperazine but not by other antipsychotics. Eur. J. Pharmacol. 256, 211-214 (1994).

13 Terai, M., Usuda, S., Kuroiwa, I., Noshiro, O. \& Maeno, H. Selective binding of YM-09151-2, a new potent neuroleptic, to D2-dopaminergic receptors. Jpn. J. Pharmacol. 33, 749-755 (1983).

14 Petrow, V. \& Stephenson, O. Analgesics. III. Salicylamide derivatives. J. Pharm. Pharmacol. 10, 96-102 (1958).

15 Justin-Besancon, L. \& Laville, C. Antiemetic action of metoclopramide with respect to apomorphine and hydergine. C. R. Seances. Soc. Biol. Fil. 158, 723-727 (1964).

16 Iwanaga, Y., Miyashita, N., Saito, T., Morikawa, K. \& Itoh, Z. Gastroprokinetic effect of a new benzamide derivative itopride and its action mechanisms in conscious dogs. Jpn. J. Pharmacol. 71, 129-137 (1996).

17 Black, S. \& Wright, N. G. Aspartic beta-semialdehyde dehydrogenase and aspartic betasemialdehyde. J. Biol. Chem. 213, 39-50 (1955).

18 Patterson, M. K. Jr. \& Orr, G. L-Asparagine biosynthesis by nutritional variants of the Jensen sarcoma. Biochem. Biophys. Res. Commun. 26, 228-233 (1967).

19 Milman, H. A. \& Cooney, D. A. Partial purification and properties of L-asparagine synthetase from mouse pancreas. Biochem. J. 181, 51-59 (1979).

20 Hongo, S. \& Sato, T. Some molecular properties of asparagine synthetase from rat liver. Biochim. Biophys. Acta. 742, 484-489 (1983).

21 Britton, H. T. S. \& Robinson, R. A. Universal buffer solutions and the association constant of veronal. J. Chem. Soc. 1456-1462 (1931).

Supplementary Information accompanies the paper on The Journal of Antibiotics website (http://www.nature.com/ja) 